Schmerz 2022 $\cdot 36: 357-362$

https://doi.org/10.1007/s00482-021-00598-7

Eingegangen: 25. Februar 2021

Überarbeitet: 30 . August 2021

Angenommen: 21. September 2021

Online publiziert: 3. November 2021

(ㅇ) Der/die Autor(en) 2021

\section{Bedarf und Vorhersagbarkeit von Magnetresonanztomographie- untersuchungen bei Patienten mit implantiertem Neurostimulator}

\author{
Marco Reining ${ }^{1}$ Dirk Winkler ${ }^{2}$. Joachim Böttcher ${ }^{3}$ Jürgen Meixensberger ${ }^{2}$. \\ Michael Kretzschmar ${ }^{1,4}$ \\ ' Klinik für Schmerz- und Palliativmedizin, SRH Wald-Klinikum Gera GmbH, Gera, Deutschland \\ ${ }^{2}$ Klinik und Poliklinik für Neurochirurgie, Universitätsklinikum Leipzig, Leipzig, Deutschland \\ ${ }^{3}$ Friedrich-Schiller-Universität Jena, Jena, Deutschland \\ ${ }^{4}$ Campus Gera, SRH Hochschule für Gesundheit, Gera, Deutschland
}

Hintergrund: Bei steigender Zahl von Magnetresonanztomographie(MRT)Untersuchungen in der deutschen Gesamtbevölkerung gibt es keine Daten zum Untersuchungsbedarf von Patienten mit implantiertem Neurostimulator in Deutschland. Publizierte Daten aus den USA legen einen hohen Bedarf nahe. Die eingeschränkte MRT-Zulassung der Implantate ist in der täglichen Praxis ein häufiges Problem.

Ziel: Im Fokus steht der MRT-Bedarf dieser Schmerzpatienten und die Vorhersagbarkeit zum Zeitpunkt der Implantation.

Material und Methoden: Es erfolgte eine retrospektive Auswertung der Datenbank unseres Klinikinformationssystems. Gesucht wurden alle im Zeitraum November 2011 bis März 2019 in unserem Klinikum angeforderten MRT-Untersuchungen für Patienten mit implantiertem Neurostimulator. Zudem erfolgte ein Abgleich mit den im gleichen Zeitraum durchgeführten Implantationen derartiger Stimulationssysteme.

Ergebnisse: Es konnten 171 durchgeführte MRT-Untersuchungen und 22 Anforderungen ohne nachfolgende Untersuchung ausgewertet werden. Bei 83 von 294 Patienten, die in unserem Zentrum mit implantierten Neurostimulatoren versorgt wurden, erfolgte mindestens eine MRT-Untersuchung in unserem Klinikum. Wir beobachten eine stetig steigende Nachfrage. In 111 von 171 durchgeführten Untersuchungen (65\%) bestand kein Zusammenhang zwischen der zur Implantation führenden Indikation und der Indikation für die MRT. Eine Vorhersagbarkeit konnte nur bei 43 von 193 MRT-Anforderungen (22\%) unterstellt werden.

Diskussion: Patienten mit implantiertem Neurostimulator haben auch in Deutschland einen hohen Bedarf an MRT-Diagnostik, welcher zum Zeitpunkt der Implantation nicht vorhersagbar ist. Daher sollten ausschließlich MRT-taugliche Systeme implantiert werden. Die Industrie ist aufgefordert, die Implantate und deren Zulassungen an den Bedarf anzupassen.

\title{
Schlüsselwörter
}

Elektrische Rückenmarkstimulation · Diagnostische Bildgebung · Neuromodulation · Versorgungsforschung $\cdot$ Schmerztherapie 


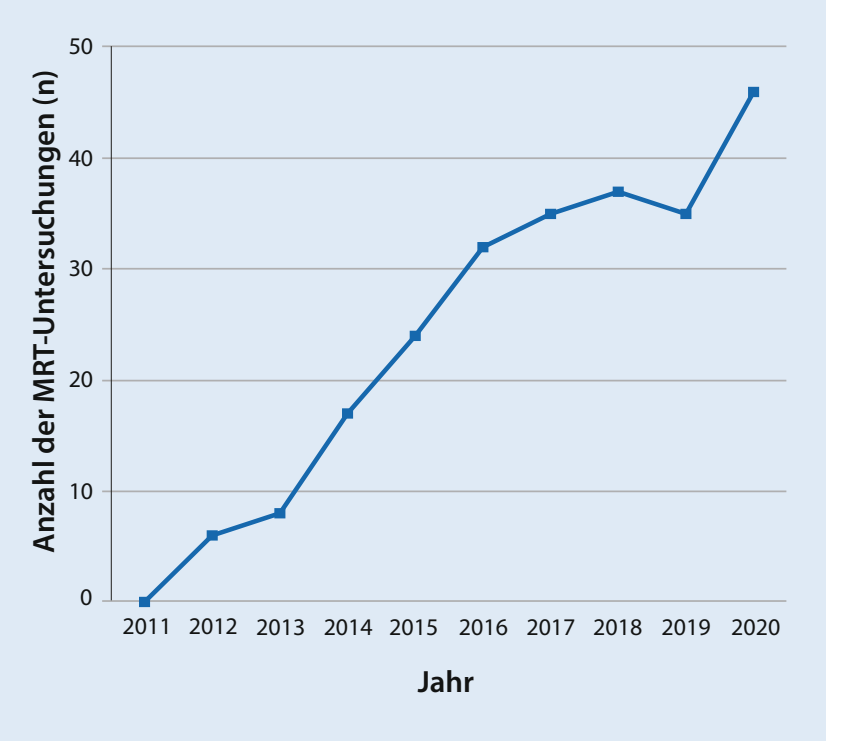

Abb. 1 ॥ Anzahl der durchgeführten MRT-Untersuchungen bei Patienten mit implantiertem Neurostimulator pro Jahr. 2019 mussten einige Untersuchungen wegen mehrfach defektem MRT-System abgesagt werden

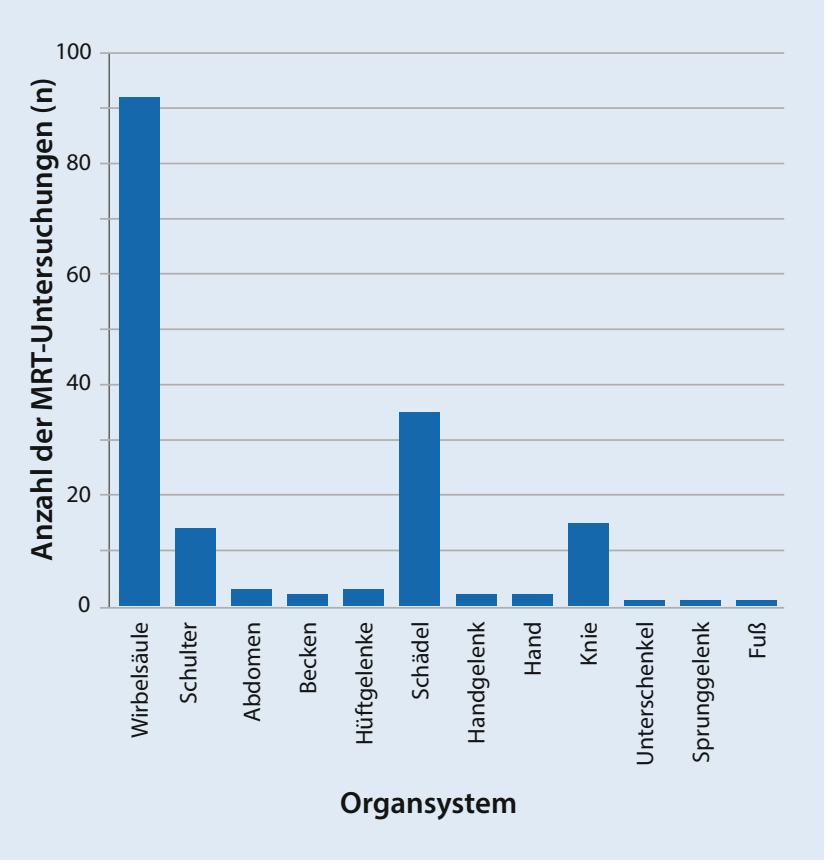

Abb. $2 \Delta$ Verteilung der MRT-Untersuchungen nach untersuchtem Organsystem. Im Sinne der Zulassung vieler Implantate gehören Untersuchungen der Schulter und der Hüftgelenke zum Rumpfbereich

\section{Hintergrund und Fragestellung}

Die Neurostimulation ist ein etabliertes Verfahren zur Behandlung neuropathischer und ischämischer Schmerzen. Ein häufiges klinisches Problem in der Nachsorge ist die fehlende oder eingeschränkte Zulassung dieser Implantate für Magnetresonanztomographie(MRT)Untersuchungen. Amerikanische Registerdaten legen einen hohen MRT-Bedarf dieser Patienten nahe, Daten aus Deutschland wurden bisher nicht publiziert. Auch die Frage nach der Vorhersagbarkeit zukünftiger MRT-Scans zum Zeitpunkt der Implantation ist bisher nicht beantwortet, dies könnte aber eine Rolle bei der Wahl der Implantate spielen.

Die Magnetresonanztomographie hat sich aufgrund des exzellenten Weichteilkontrasts bei vielen Erkrankungen zum diagnostischen Verfahren der ersten Wahl entwickelt [1]. Die Bedeutung der MRT belegen auch stetig steigende Untersuchungszahlen weltweit, in Deutschland stieg die Zahl der MRT-Untersuchungen pro 1000 Einwohner von 97,0 im Jahr 2009 auf geschätzt 145,1 im Jahr 2018 [15].

Gleichzeitig sind die elektrische Rückenmarkstimulation („spinal cord stimulation“
[SCS]), die Hinterwurzelganglionstimulation („dorsal root ganglion stimulation" [DRG-S]), die periphere Nervenstimulation (PNS) und die periphere Nervenfeldstimulation (PNFS) etablierte invasive Verfahren zur Therapie chronischer Schmerzen [5, 8, 11]. Im Jahr 2019 wurden in Deutschland 1954 Neurostimulatoren zur rückenmarksnahen Stimulation (SCS und DRGS) implantiert [21]. Daten zur Prävalenz in der deutschen Gesamtbevölkerung sind nicht verfügbar, wir schätzen, dass derzeit mindestens 10.000 Patienten mit einem Neurostimulator versorgt sind. Aktuelle deutsche Leitlinien zur elektrischen Rückenmarkstimulation empfehlen eine Behandlung insbesondere bei persistierenden Schmerzen nach Wirbelsäuleneingriffen („failed back surgery syndrome" [FBSS]), komplexem regionalem Schmerzsyndrom (CRPS) Typ I und II, ischämischen Schmerzen bei Angina pectoris und peripherer arterieller Verschlusserkrankung (pAVK) und sonstigen neuropathischen Schmerzen [7, 8].

Die meisten derzeit verfügbaren Generatoren haben eine sehr limitierte $\mathrm{Zu}$ lassung für MRT-Untersuchungen mit vielen Einschränkungen (,MR conditional“), älteren Modellen fehlt häufig die MRT-Zu- lassung. Hinsichtlich des Zulassungsstatus muss immer das Gesamtsystem mit allen Komponenten betrachtet werden: ein zugelassener Generator und eine zugelassene Elektrode bedeuten nicht automatisch eine Zulassung des Gesamtsystems. Die Einschränkungen können z. B. die Art und Lage der Elektroden, die zu untersuchenden Körperregionen, spezifische Anforderungen an das MRT-System (z. B. geschlossene horizontale Systeme, Magnetfeldstärke) und nutzbare Spulen betreffen. Zusätzlich gelten Grenzen für die technischen Parameter (z.B. spezifische Absorptionsrate, Gradientenfelder, Untersuchungsdauer). Auskunft hierzu geben die Bedienungsanleitungen oder einschlägige Übersichtsarbeiten [18, 19]. Aufgrund dieser vielfältigen Einschränkungen sind nur wenige MRT-Untersuchungen innerhalb der Zulassung möglich [17].

Bisher gibt es nur zwei Arbeiten, welche sich auf Basis einer kommerziellen USamerikanischen Datenbank (Truven Reuters MarketScan ${ }^{\circledR}$ Database, Truven Health Analytics, Ann Arbor, MI, USA) mit dem Bedarf an MRT-Untersuchungen für diese Patientengruppe beschäftigen: Desai und Kollegen schätzen, dass $82-84 \%$ der mit einem Neurostimulator versorgten Patien- 


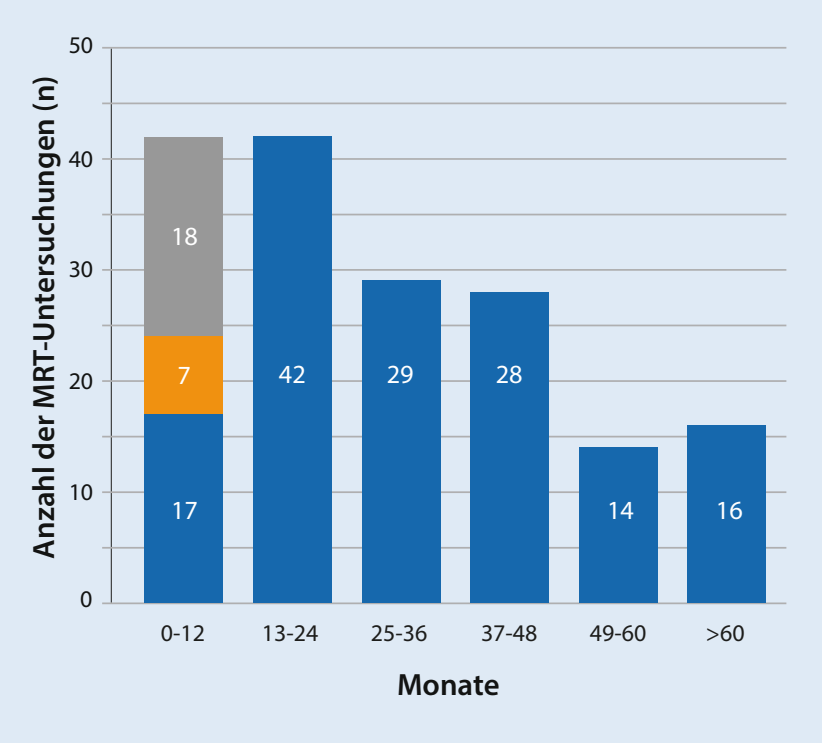

Abb. $3 \triangleleft$ Anzahl der Untersuchungen in Abhängigkeit von der seit der Implantation des Neurostimulators vergangenen Zeit. Der erste Balken ist dreigeteilt: blau 0-3 Monate, orange 3-6 Monate und grau 7-12 Monate nach der Implantation des Neurostimulators ten innerhalb der ersten fünf Jahre mindestens eine MRT-Untersuchung benötigen [6]. Farber und Kollegen resümieren, dass Patienten mit FBSS, welches die häufigste Indikation zur Neurostimulation ist, einen im Vergleich mit der Durchschnittsbevölkerung 1,73-fachen MRT-Bedarf haben. Patienten mit einem Neurostimulator erhalten aber nur halb so viele MRTUntersuchungen wie Patienten ohne Neurostimulator [9].

Diese Arbeit soll zwei Fragen beantworten:

- Wie hoch ist der MRT-Bedarf von

Patienten mit implantiertem Neurostimulator in Deutschland?

- Ist es möglich, den zukünftigen MRTBedarf zum Zeitpunkt der Implantation des Neurostimulators vorherzusagen?

\section{Materialien und Methoden}

\section{Datenakquise}

Nach positivem Ethikvotum erfolgte eine Suche in der Datenbank unseres Klinikinformationssystems nach MRT-Anforderungen und gleichzeitig bestehenden Hinweisen auf das Vorhandensein eines Neurostimulators (Schlüsselwörter, Diagnose- bzw. Prozedurenschlüssel, Kontakt mit unserer Abteilung) für den Zeitraum von November 2011 (Gründung unseres Schmerzzentrums) bis März 2019. Bei allen Suchergebnissen überprüften wir die gesamte
Patientenakte und extrahierten daraus die benötigten Daten.

Zusätzlich erfolgte eine weitere Suche in der Datenbank für den gleichen Zeitraum nach allen Operationen im Zusammenhang mit einem Neurostimulator über die Prozedurenschlüssel (5-039.*). Hier wurde die Patientenakte des jeweiligen Aufenthalts überprüft und die notwendigen Daten extrahiert.

Alle Zuordnungen zu Gruppen erfolgten durch den Erstautor und wurden durch den Letztautor überprüft, Fälle mit differenter Einschätzung wurden im gesamten Autorenteam diskutiert und die $\mathrm{Zu}$ ordnung festgelegt.

\section{Statistik}

Zur Datenerfassung und -analyse verwendeten wir eine handelsübliche Tabellenkalkulationssoftware mit allen aktuellen Updates (Microsoft Excel ${ }^{\circledR}$ 365, 64 Bit; Microsoft, Inc., Redmond, WA, USA).

Es erfolgte eine Beratung durch einen Statistiker, dieser empfahl eine rein deskriptive Darstellung unserer Daten.

\section{Ergebnisse}

\section{Identifikation und Charakterisierung} der Studienteilnehmer

Die Suche nach MRT-Anforderungen in der Datenbank unseres Klinikinformationssystems lieferte 1024 Treffer, in 193 Fällen konnte das Vorhandensein eines implantierten Neurostimulators bestätigt werden. In 22 Fällen erfolgte aus verschiedenen Gründen keine MRT-Untersuchung, sodass 171 durchgeführte MRT-Untersuchungen und 22 Anforderungen ohne resultierende MRT-Untersuchung ausgewertet werden konnten.

Die 171 MRT-Untersuchungen betrafen 100 verschiedene Patienten (49 Männer, 51 Frauen) im Alter von 28 bis 87 Jahren (Median: 57 Jahre). Häufigste Indikationen für die Implantation des Neurostimulators waren ein FBSS $(n=49)$, Wirbelsäulenbeschwerden ohne vorangegangene Operation $(n=14)$, CRPS $(n=9)$ und periphere Nervenschäden $(n=9)$.

Die Suche nach den durchgeführten Operationen im Zusammenhang mit Neurostimulatoren ergab 1065 Treffer, hiervon betreffen 294 Eingriffe die Erstimplantation eines Neurostimulators.

\section{Charakterisierung des MRT-Bedarfs}

Wir beobachten in unserer Einrichtung eine stetig zunehmende Zahl von MRTUntersuchungen bei Patienten mit implantiertem Neurostimulator (vgl. - Abb. 1). Die untersuchten Körperregionen sind in - Abb. 2 dargestellt. 121 der 171 durchgeführten MRT-Untersuchungen (70,8\%) können dem Fachgebiet Orthopädie/ Unfallchirurgie einschließlich Wirbelsäulenchirurgie zugeordnet werden, weitere 37 Untersuchungen (21,6\%) sind dem Fachgebiet Neurologie zuzuordnen. 8 Fälle $(4,7 \%)$ betrafen onkologische Fragestellungen, weitere Anforderungen stammen aus den Fachgebieten Hals-Nasen-OhrenHeilkunde $(n=2)$, Endokrinologie $(n=2)$ und Psychiatrie $(n=1)$.

Wie bereits oben beschrieben, wurde die Erstimplantation eines Neurostimulators bei 294 Patienten in unserem Schmerzzentrum vorgenommen. 84 dieser 294 Patienten (28,6\%) benötigten mindestens eine MRT-Untersuchung, insgesamt wurden 141 MRT-Untersuchungen an in unserem Klinikum implantierten Patienten durchgeführt. Die übrigen $30 \mathrm{MRT}$ Untersuchungen verteilen sich auf 16 extern implantierte Patienten, welche bei uns wegen Schließung der implantierenden Einrichtung $(n=20)$, Wechsel des Wohnorts $(n=1)$ oder Neueröffnung 
Tab. 1 Handelt es sich bei der Indikation zur MRT-Diagnostikum eine akute oder eine chronische Erkrankung? $(n=193)$

\begin{tabular}{|l|l|}
\hline- & $n(\%)$ \\
\hline Neues akut aufgetretenes gesundheitliches Problem & $87(45,1)$ \\
\hline Akute Verschlechterung einer vorbestehenden chronischen Erkrankung & $46(23,8)$ \\
\hline Chronische Erkrankung allein & $60(31,1)$
\end{tabular}

einer näher am Wohnort gelegenen Einrichtung $(n=2)$ weiterbetreut werden. Hinzu kommen Patienten, welche im implantierenden externen Zentrum betreut werden, aber zum Zeitpunkt der MRT-Untersuchung in unserem Klinikum stationär behandelt wurden $(n=7)$.

Wir haben beispielhaft die Untersuchungszahlen für das Kalenderjahr 2018 (letztes vollständiges Kalenderjahr im Beobachtungszeitraum) näher analysiert: Es wurden 37 MRT-Untersuchungen an 29 verschiedenen Patienten durchgeführt, davon wurden 3 Patienten mit 5 MRTUntersuchungen nicht in unserem Haus implantiert. Seit der Gründung unseres Zentrums im Jahr 2011 bis zum Jahresende 2018 haben wir insgesamt 288 Patienten einen Neurostimulator erstmals implantiert. Im Jahr 2018 erhielten somit 9,0\% der bei uns mit einem Neurostimulator versorgten Patienten mindestens eine MRT-Untersuchung.

In 93 von 171 Fällen (54,4\%) konnten für die weitere Behandlung entscheidende Befunde erhoben werden. Neben neuen, wegweisenden Diagnosen zählten hierzu auch Negativbefunde, zum Beispiel in der Tumornachsorge oder beim Ausschluss einer klinisch gut begründeten Verdachtsdiagnose. Ein konkretes Beispiel für die zuletzt genannte Konstellation ist der Ausschluss einer Spondylodiszitis bei Rückenschmerzen, Fieber und massiv erhöhten Entzündungswerten im Labor. In 72 Fällen $(42,1 \%)$ konnten keine therapieverändernden Befunde erhoben werden, und in 6 Fällen $(3,5 \%)$ war aufgrund frühzeitiger Untersuchungsabbrüche keine Befundung möglich.

Die Zeit zwischen Implantation des Neurostimulators und der MRT-Untersuchung ist in - Abb. 3 dargestellt, der Median beträgt 25 Monate (Spannweite: 0-119 Monate). In 4 Fällen war die MRTUntersuchung bereits nach Implantation der Elektrode, aber noch vor Implantation des Impulsgenerators erforderlich; diese Fälle haben wir mit 0 Monaten berücksichtigt, bei Ausschluss dieser Fälle ergibt sich ein Median von 27 Monaten bei gleicher Spannweite. In einem Fall ist das genaue Datum der Implantation bei extern erfolgtem Eingriff nicht mehr nachvollziehbar; dieser Patient gehört eindeutig in die Gruppe > 60 Monate und hat daher keinen Einfluss auf die Qualität unserer Daten und deren Auswertung.

\section{Vorhersagbarkeit von MRT- Untersuchungen}

Es wurden alle 193 MRT-Anforderungen ausgewertet. Die retrospektive Analyse der Vorhersagbarkeit stützte sich auf 3 Fragen:

- Ein Zusammenhang zwischen der Indikation zur Implantation des Neurostimulators und der Indikation für die MRT-Untersuchung konnte nur in 70 von 193 Fällen $(26,3 \%)$ unterstellt werden.

- Die Unterteilung in akute oder chronische Erkrankungen ist in $\mathbf{0}$ Tab. 1 dargestellt.

- Die zur MRT-Diagnostik führende chronische Erkrankung war in 58 von 106 Fällen (54,7\%) zum Zeitpunkt der Implantation des Neurostimulators bekannt.

Letztendlich können wir nur in 43 Fällen $(22,3 \%)$ eine Vorhersehbarkeit der Notwendigkeit einer MRT-Diagnostik unterstellen. Diese betrafen:

- 29 Patienten mit chronischen Rückenbeschwerden: Durch die behandelnden Fachärzte wurde die eindeutige, kaum widerlegbare Indikation zu Kontrolluntersuchungen gestellt.

- 12 Patienten mit vorbestehenden chronischen Erkrankungen mit absehbarem Bedarf an Kontrolluntersuchungen (z. B. onkologische Patienten, zerebrale Gefäßmalformationen).
- Je 1 Patient mit akuter Verletzung und mit akuter Exazerbation einer vorbestehenden chronischen Erkrankung.

\section{Diskussion}

\section{MRT-Bedarf von Patienten mit implantiertem Neurostimulator}

Mit dieser retrospektiven Arbeit publizieren wir erstmals Daten zum MRT-Bedarf von Patienten mit implantiertem Neurostimulator in Deutschland. Wir konnten zeigen, dass ca. $29 \%$ der in unserem Klinikum implantierten Patienten im Betrachtungszeitraum von ca. $7 \frac{1}{2}$ Jahren mindestens eine MRT-Untersuchung in unserem Klinikum erhalten haben. Wir haben deutliche Anhaltspunkte, dass unsere Zahlen den MRT-Bedarf dieser Patientengruppe unterschätzen. Zum einen erhalten wir regelmäßig Anfragen aus anderen Kliniken zur MRT-Zulassung unserer Implantate, da dort die Indikation zur MRT-Untersuchung gestellt wurde. Zum anderen berichten uns unsere Patienten immer wieder von extern erfolgten MRT-Untersuchungen.

Im Vergleich mit den publizierten USamerikanischen Registerdaten [6, 9], nach denen 82-84\% der Patienten mit Neurostimulator eine MRT innerhalb von 5 Jahren benötigen, erscheint der MRT-Bedarf unserer Patienten geringer. In Deutschland erhielten etwa 7,19\% der Bevölkerung im Jahr 2009 mindestens eine MRT-Untersuchung [2]. Von 2009 bis 2018 ist die Zahl der MRT-Untersuchungen pro 1000 Einwohner in Deutschland um ca. $50 \%$ gestiegen [15], somit ist zu erwarten, dass 10-11\% der Bevölkerung mindestens eine MRTUntersuchung im Jahr 2018 hatten. Auch wir beobachten bei unseren Patienten eine deutliche Zunahme an durchgeführten MRT-Untersuchungen, im Jahr 2018 erhielten 9,0\% unserer Patienten mindestens eine MRT-Untersuchung; dies entspricht nahezu dem MRT-Bedarf der deutschen Durchschnittsbevölkerung, wobei extern erfolgte MRT-Untersuchungen unberücksichtigt bleiben.

Bei der Interpretation unserer Daten muss die stark eingeschränkte Zulassung der Neurostimulationssysteme für MRTUntersuchungen berücksichtigt werden [19]. 66,7\% der bei uns durchgeführten MRT-Untersuchungen betreffen den 
Rumpfbereich, welcher in Bezug auf die MRT-Zulassung der Implantate häufig problematisch ist. In vielen Fällen ist die Untersuchung nicht durch die Zulassung gedeckt, sodass zunächst die zugelassenen nichtinvasiven Verfahren der zweiten Wahl (Röntgen, Computertomographie, Sonographie) ausgeschöpft wurden und häufig auf die eigentlich indizierte MRTUntersuchung aufgrund der fehlenden Zulassung verzichtet wurde. Farber und Kollegen beschreiben einen hohen Bedarf an Bildgebung und einen geringeren Anteil an MRT-Bildgebung [9], diese Beobachtung können wir grundsätzlich bestätigen. Studien zur Sicherheit von MRTUntersuchungen sind rar und beziehen sich mit unterschiedlicher Schwerpunktsetzung zumeist auf ältere, heute nur noch selten anzutreffende Implantate [4, $13,14,17,22]$.

Die stark eingeschränkte Zulassung führt zu einem Mehraufwand beim Radiologen: Häufig müssen am MRT-Gerät spezielle Einstellungen der technischen Parameter entsprechend den Empfehlungen der Hersteller vorgenommen werden, welche zuvor aufwendig recherchiert werden müssen. Dies führt dazu, dass diese Untersuchungen vielfach einen deutlich höheren Zeitaufwand erfordern, welcher schlecht mit den häufig eng getakteten Terminplanungen in Einklang zu bringen ist. Zudem bestehen bei vielen Radiologen Bedenken hinsichtlich der Patientensicherheit. Daher ist es nach wie vor nicht ohne Weiteres möglich, diese Patienten einer solchen Diagnostik zuzuführen.

In unserer Kohorte führte die MRTUntersuchung bei $54,4 \%$ der Fälle zu eindeutig therapierelevanten Befunden. Der Anteil relevanter Befunde variiert stark abhängig vom untersuchten Organsystem, der Fragestellung, Indikationsstellung und der Verfügbarkeit von MRT-Diagnostik. Daher ist ein Vergleich dieses Parameters bei unserem selektierten Patientengut mangels passender Vergleichsgruppen nicht möglich. Zudem kann auch der Ausschluss einer potenziell gefährlichen Erkrankung auf den zweiten Blick therapierelevant sein, sodass dieser Zahlenwert kein geeigneter Qualitätsparameter ist.

Im Vergleich zur Durchschnittsbevölkerung ist der hohe Anteil an Untersuchungen der Wirbelsäule auffällig [2]. Dies ist si- cherlich durch die Selektion des Patientenguts zu erklären. Ebenfalls überraschend ist der hohe MRT-Bedarf in den ersten 2,5 Jahren nach der Erstimplantation eines Neurostimulators, hierfür haben wir derzeit noch keine plausible Erklärung.

\section{Vorhersagbarkeit künftiger MRT- Untersuchungen}

Zur Frage der Vorhersagbarkeit zukünftig erforderlicher MRT-Untersuchungen zum Zeitpunkt der Implantation des Neurostimulators gibt es nach kritischer Durchsicht der verfügbaren Literatur noch keine Arbeiten.

Ein Großteil $(63,7 \%)$ der erforderlichen MRT-Untersuchungen steht in keinem Zusammenhang mit der (Schmerz-)Indikation für den Neurostimulator. Zumeist nicht vorhersehbare neue akute Ereignisse $(45,1 \%)$ und auch akute Ereignisse bei vorbestehenden chronischen Erkrankungen $(23,8 \%)$ sind für einen überwiegenden Teil der Untersuchungen verantwortlich. Die chronischen Erkrankungen waren nur in $54,7 \%$ der Fälle zum Zeitpunkt der Implantation bereits bekannt. Bei zum Zeitpunkt der Implantation noch nicht bekannten Erkrankungen kann die Vorhersagbarkeit ebenfalls eindeutig verneint werden.

Retrospektiv waren nur 22,3\% der MRT-Anforderungen vorhersehbar, eine prospektive Einschätzung des zukünftigen MRT-Bedarfs vor der Implantation erscheint deutlich schwieriger. Daher sollte aus unserer Sicht immer ein Neurostimulator mit möglichst umfassender MRT-Zulassung implantiert werden. Ein besonderer Bedarf liegt in der Bildgebung des Rumpfbereichs. Die Hersteller der Implantate sind aufgefordert, bei künftigen Produktentwicklungen diesen MRT-Bedarf zu berücksichtigen und die Implantate bzw. deren MRT-Zulassung zu verbessern. Wünschenswert ist eine Untersuchung des gesamten Körpers im Normalmodus des MRT-Geräts (spezifische Absorptionsrate $\leq 2 \mathrm{~W} / \mathrm{kg}$ ) unabhängig von der Platzierung der Elektroden. Zukünftig wird aufgrund besserer Bildqualität auch die Zulassung für Untersuchungen in 3-Tesla-MRT-Geräten eine Rolle spielen [20], für einige Indikationen konnte bereits ein Vorteil der Bildgebung mit höherer Feldstärke gezeigt werden $[3,10,12,16]$.
Wesentliche Limitation unserer Arbeit ist das monozentrische, retrospektive und primär auf die Untersuchung der Sicherheit dieser MRT-Untersuchungen ausgelegte Studiendesign.

Fazit für die Praxis

- Patienten mit implantiertem Neurostimulator haben einen relevanten MRT-Bedarf.

- Die MRT-Untersuchungen erfolgen häufig kurz nach der Implantation und betreffen überwiegend den Rumpf (inklusive Wirbelsäule).

- Der künftige Bedarf an MRT-Untersuchungen ist zum Zeitpunkt der Implantation in der überwiegenden Zahl der Fälle nicht vorhersehbar.

- Daher sollten ausschließlich MRT-kompatible Neurostimulatoren implantiert werden.

- Die Hersteller sind aufgefordert, die Implantate und deren MRT-Zulassung an die klinischen Bedürfnisse anzupassen und um die Verwendung in MRT-Geräten mit 3-Tesla-Technik zu erweitern.

\section{Korrespondenzadresse}

\section{Marco Reining}

Klinik für Schmerz- und Palliativmedizin, SRH

Wald-Klinikum Gera GmbH

Straße des Friedens 122, 07549 Gera,

Deutschland

Marco.Reining@srh.de

Danksagung. Die Autoren danken dem Statistischen Bundesamt (Destatis) für die kostenlose Überlassung von Auszügen der DRG-Statistik. Zudem danken wir Herrn Prof. Dr. Marcus Schwarz von der SRH Hochschule für Gesundheit, Campus Gera, für die Beratung zur statistischen Auswertung.

\section{Einhaltung ethischer Richtlinien}

Interessenkonflikt. M. Reining und M. Kretzschmar sind als Prüfärzte an Studien der Firma Abbott Laboratories beteiligt. Die Studien wurden durch das Studienzentrum des SRH Wald-Klinikums Gera betreut, alle Honorare wurden vom Studienzentrum in Rechnung gestellt und an das SRH Wald-Klinikum Gera gezahlt. D. Winkler, J. Böttcher und J. Meixensberger erklären, dass kein Interessenkonflikt besteht.

Für diesen Beitrag wurden von den Autoren keine Studien an Menschen oder Tieren durchgeführt. Für die aufgeführten Studien gelten die jeweils dort angegebenen ethischen Richtlinien. Diese retrospektive Studie erfolgte nach Konsultation der zuständigen Ethikkommission der Landesärztekammer Thüringen (Bescheid vom 07.05.2019, Registrierungsnummer 64752/2019/28) und im Einklang mit nationalem Recht. 
Open Access. Dieser Artikel wird unter der Creative Commons Namensnennung 4.0 International Lizenz veröffentlicht, welche die Nutzung, Vervielfältigung, Bearbeitung, Verbreitung und Wiedergabe in jeglichem Medium und Format erlaubt, sofern Sie den/die ursprünglichen Autor(en) und die Quelle ordnungsgemäß nennen, einen Link zur Creative Commons Lizenz beifügen und angeben, ob Änderungen vorgenommen wurden.

Die in diesem Artikel enthaltenen Bilder und sonstiges Drittmaterial unterliegen ebenfalls der genannten Creative Commons Lizenz, sofern sich aus der Abbildungslegende nichts anderes ergibt. Sofern das betreffende Material nicht unter der genannten Creative Commons Lizenz steht und die betreffende Handlung nicht nach gesetzlichen Vorschriften erlaubt ist, ist für die oben aufgeführten Weiterverwendungen des Materials die Einwilligung des jeweiligen Rechteinhabers einzuholen.

Weitere Details zur Lizenz entnehmen Sie bitte der Lizenzinformation auf http://creativecommons.org/ licenses/by/4.0/deed.de.

\section{Literatur}

1. American College of Radiology (2020) January 2020 ACR appropriateness criteria. https://www. acr.org/Clinical-Resources/ACR-AppropriatenessCriteria.Zugegriffen: 18. Nov. 2020

2. Barmer Gek (Hrsg) (2011) Barmer GEK Arztreport 2011, Schwerpunkt: Bildgebende Diagnostik. Asgard, St. Augustin, S202-215

3. Crespo-Rodríguez AM, De Lucas-Villarrubia JC, Pastrana-Ledesma M et al (2017) The diagnostic performance of non-contrast 3-Tesla magnetic resonance imaging (3-T MRI) versus 1.5-Tesla magnetic resonance arthrography (1.5-T MRA) in femoro-acetabular impingement. Eur J Radiol 88:109-116

4. De Andres J, Valia JC, Cerda-Olmedo G et al (2007) Magnetic resonance imaging in patients with spinal neurostimulation systems. Anesthesiology 106:779-786

5. DeerTR,Grider JS, LamerTJetal (2020) Asystematic literature review of spine neurostimulation therapies for the treatment of pain. Pain Med 21:1421-1432

6. Desai MJ, Hargens LM, Breitenfeldt MD et al (2015) The rate of magnetic resonance imaging in patients with spinal cord stimulation. Spine 40:E531-E537

7. Dgai, Dga, Dgk, Dgnc, Dgnm, Dgn, Dgpsf, Dgss S3-Leitlinie Epidurale Rückenmarkstimulation zur Therapie chronischer Schmerzen - Langfassung. Version 7/2013. https://www.awmf.org/leitlinien/ detail/ll/008-023.html.Zugegriffen:6. Mai 2020

8. Dgs (Hrsg) (2019) DGS-Praxisleitlinie Epidurale Rückenmarkstimulation zur Therapie chronischer Schmerzen

9. Farber SH, Han JL, Petraglia lii FW et al (2017) Increasing rates of imaging in failed back surgery syndrome patients: implications for spinal cord stimulation. Pain Physician 20:E969-E977

10. Fukushima K, Miyazaki I, Kobayashi K et al (2016) Optimal imaging parameters and the advantage of renal artery image using time-spatial labeling inversion pulse at 3 tesla magnetic resonance imaging: comparison of image quality for 1.5 tesla magnetic resonance imaging. Nihon Hoshasen Gijutsu Gakkai Zasshi 72:1113-1121

\section{Need for and predictability of magnetic resonance imaging examinations in patients with implanted neurostimulators}

Background: With an increasing number of magnetic resonance imaging (MRI) examinations in the general population, there are no data available regarding the requirements of patients with implanted neurostimulators in Germany. Published data from the United States of America suggest a high need. The limited approval for MRI scans of implants are a common problem.

Objective: The focus is on the MRI needs of these pain patients and the predictability at the time of implantation.

Material and method: We carried out a retrospective evaluation of the database of our hospital information system. We searched for all MRI requests for patients with an implanted neurostimulator between November 2011 and March 2019. In addition, we compared these data with the implantation of neurostimulators in the same period. Results: We identified $171 \mathrm{MRI}$ examinations and 22 requests without a subsequent examination. Out of 294 (28\%) patients implanted in our center 83 had at least $1 \mathrm{MRI}$ scan in our hospital. We observed a steadily increasing demand. In 111 of 171 (65\%) performed examinations, there was no association between the indications leading to neurostimulator implantation and to MRI. A predictability could only be assumed for 43 of $193(22 \%)$ MRI requests.

Conclusion: In Germany, patients with an implanted neurostimulator have a high need for MRI diagnostics which cannot be predicted at the time of implantation. Therefore, only MRI-conditional systems should be implanted. The manufacturers need to adapt the implants and their approval to requirements.

\section{Keywords}

Spinal cord stimulation · Diagnostic imaging · Neuromodulation · Health services research · Pain medicine

11. Hofmeister M, Memedovich A, Brown Set al (2020) Effectiveness of neurostimulation technologies for the management of chronic pain: a systematic review. Neuromodulation 23:150-157

12. Kosior RK, Wright CJ, Kosior JC et al (2007) 3-Tesla versus 1.5-Tesla magnetic resonance diffusion and perfusion imaging in hyperacute ischemic stroke. Cerebrovasc Dis 24:361-368

13. Manfield J, Bartlett R, Park N (2019) Safety and utility of spinal magnetic resonance imaging in patients with high-frequency spinal cord stimulators: a prospective single-centre study. Stereotact Funct Neurosurg 97:272-277

14. Mutter UM, Bellut D, Porchet F et al (2013) Spinal magnetic resonance imaging with reduced specific absorption rate in patients harbouring a spinal cord stimulation device-A single-centre prospective study analysing safety, tolerability and image quality. Acta Neurochir 155:2327-2332

15. Organisation for Economic Co-Operation and Development (2020) Magnetic resonance imaging (MRI) exams (indicator). https://data.oecd.org/ healthcare/magnetic-resonance-imaging-mriexams.htm. Zugegriffen: 16. März 2020

16. Rahbar H, Partridge SC, Demartini WB et al (2013) Clinical and technical considerations for high quality breast MRI at 3 Tesla. J Magn Reson Imaging 37:778-790

17. Reining $M$, Winkler $D$, Boettcher J et al (2021) Magnetic resonance imaging (MRI) scans in patients with dorsal root ganglion stimulation. Pain Pract. https://doi.org/10.1111/papr.13067

18. Rubino S, Adepoju A, Kumar V et al (2016) MRI conditionality in patients with spinal cord stimulation devices. Stereotact Funct Neurosurg 94:254-258
19. Sayed D, Chakravarthy K, Amirdelfan Ket al (2020) A comprehensive practice guideline for magnetic resonance imaging compatibility in implanted neuromodulation devices. Neuromodulation 23:893-911

20. Schmitt F, Grosu D, Mohr C et al (2004) 3 Tesla MRI: successful results with higher field strengths. Radiologe 44:31-47

21. Statistisches Bundesamt (2020) Fallpauschalenbezogene Krankenhausstatistik (DRG-Statistik) 2019

22. Tronnier VM, Staubert A, Hahnel S et al (1999) Magnetic resonance imaging with implanted neurostimulators: an in vitro and in vivo study. Neurosurgery 44:118-125 (discussion 125-116) 